\title{
Prognostic value of T1 substaging on oncological outcomes in patients with non-muscle-invasive bladder urothelial carcinoma: a systematic literature review and meta-analysis
}

\author{
Mehdi Kardoust Parizi ${ }^{1,2}$. Dmitry Enikeev ${ }^{3} \cdot$ Petr V. Glybochko $^{3} \cdot$ Veronika Seebacher $^{4}$. Florian Janisch ${ }^{1,5}$. \\ Harun Fajkovic ${ }^{1}$. Piotr L. Chłosta ${ }^{6} \cdot$ Shahrokh F. Shariat $^{1,3,7,8}$
}

Received: 17 May 2019 / Accepted: 28 August 2019 / Published online: 6 September 2019

(c) The Author(s) 2019

\begin{abstract}
Purpose To evaluate the prognostic value of substaging on oncological outcomes in patients with T (or pT1) urothelial carcinoma of the bladder.

Methods A literature search using PubMed, Scopus, Web of Science, and Cochrane Library was conducted on March 2019 to identify relevant studies according to the Preferred Reporting Items for Systematic Review and Meta-analysis (PRISMA) guidelines. The pooled disease recurrence (DR) and disease progression (DP) rate in T1(or pT1) patients were calculated using a fixed or random effects model.

Results Overall 36 studies published between 1994 and 2018 including a total of 6781 bladder cancer patients with T1(or pT1) stage were selected for the systematic review and meta-analysis. Twenty-nine studies reported significant association between tumor infiltration depth or muscularis mucosa $(\mathrm{MM})$ invasion and oncological outcomes. Totally 12 studies were included in the meta-analysis. MM invasion $(\mathrm{T} 1 \mathrm{a} / \mathrm{b} / \mathrm{c}$ [or $\mathrm{pT} 1 \mathrm{a} / \mathrm{b} / \mathrm{c}$ ] or $\mathrm{T} 1 \mathrm{a} / \mathrm{b}[$ or $\mathrm{pT} 1 \mathrm{a} / \mathrm{b}$ ] substaging system) was associated with DR (pooled HR: 1.23, 95\%CI: 1.01-1.49) and DP (pooled HR: 2.61, 95\%CI: 1.61-4.23). Tumor infiltration depth (T1 m/e [or pT1 m/e] substaging system) was also associated with DR (pooled HR: 1.49, 95\%CI: 1.11-2.00) and DP (pooled HR: 3.29 , 95\% CI: 2.39-4.51).

Conclusions T1(or pT1) substaging in patients with bladder cancer is of prognostic value as it is associated with oncologic outcomes. Inclusion of this factors into the clinical decision-making process of this heterogeneous tumor may improve outcomes, while avoiding over- and under-treatment for T1(or pT1) bladder cancer.
\end{abstract}

Keywords Staging $\cdot$ Substage $\cdot$ pT1 $\cdot$ T1 $\cdot$ Bladder cancer $\cdot$ Progression $\cdot$ Prognosis $\cdot$ Urothelial carcinoma

\section{Introduction}

T1 carcinoma of the urinary bladder is a heterogeneous disease with potentially aggressive behavior leading to lethality [1]. Indeed, despite sharing many of the genetic and epigenetic factors of muscle-invasive bladder cancer, it is classified as non-muscle invasive. Yet, patients with T1 bladder cancer have an overall mortality of $33 \%$ and a cancer-specific mortality of $14 \%$ at three years after diagnosis, suggesting that these patients have a high risk of disease progression

Shahrokh F. Shariat

sfshariat@gmail.com

Extended author information available on the last page of the article and, accordingly, require meticulous surgery, endoscopic surveillance and informed clinical decision-making [2].

The variability in the outcomes of patients with T1 bladder cancer is a result of both tumor heterogeneity and pathological staging, as well as inconsistencies in risk stratification, endoscopic resection and schedules of delivery of BCG [3]. Owing to limitations in clinical staging, patients with $\mathrm{T} 1$ bladder cancer are at risk of both under-treatment with use of BCG despite recurrence, and overtreatment with early radical cystectomy. Understanding the pathologic features of T1 bladder cancers and how they impact prognosis and, therefore, could improve risk stratification to align therapy with biological risk and clinical behavior of the individual tumor $[4,5]$. While novel prognostic features such as variant histology and lymphovascular invasion have been included 
in the clinical decision-making, more features are needed to improve our prognostic accuracy [5-7].

There is a growing evidence that tumor depth and extension could be such a feature for patients with T1(or pT1) bladder cancer $[8,9]$. To test this hypothesis, we performed a systematic review and meta-analysis to evaluate the value of T1(or pT1) substaging for predicting oncological outcomes in patients with T1(or pT1) urothelial carcinoma of the bladder. T1 and pT1 were referred to disease stage in patients who underwent trans-urethral resection of bladder tumor (TURBT) and radical cystectomy, respectively.

\section{Materials and methods}

\section{Search strategy}

A full electronic literature search using PubMed, Scopus, Web of Science, and Cochrane Library was conducted by two independent authors on March 2019 to find relevant studies for this systematic review and meta-analysis according to the Preferred Reporting Items for Systematic Review and Meta-analysis (PRISMA) guidelines [10]. The search terms used were ("T1" OR "T1a" OR "T1b" OR "T1 m" OR "T1e" OR "muscularis mucosa invasion" OR "subclassification" OR "substage" OR "substaging”) AND ("bladder cancer" OR "bladder carcinoma" OR "bladder neoplasm"). The protocol for this systematic review was registered in PROSPERO (Prospective Register of Systematic Reviews, CRD42019129661) and is available in full on the University of York website.

\section{Inclusion criteria}

The following criteria were considered to select eligible studies: prospective or retrospective studies including full text regarding T1(or pT1) substaging in patients with nonmuscle-invasive bladder cancer (NMIBC) with oncological outcomes including disease recurrence (DR) and disease progression (DP). We excluded studies in other than English, meeting abstract, case reports, review articles, replies, expert opinions, and comment letters.

\section{Data extraction}

Data were extracted on first author, year of publication, patients, region of study, recruitment period, study design, total number of T1(or pT1) patients, number of substaged $\mathrm{T} 1$ (or pT1) patients, substaging system, patients' age, and follow-up duration. Oncological outcomes including DR and DP were the primary outcomes of interest. DR was defined as histological detection of bladder cancer and DP was defined as development of muscle-invasive disease or distant metastasis after primary treatment. Two independent reviewers assessed all full text studies and excluded inappropriate ones after screening based on the study title and abstract. The muscularis mucosa (MM) invasion substaging was defined as $\mathrm{T} 1 \mathrm{a} / \mathrm{b}$ (or $\mathrm{pT} 1 \mathrm{a} / \mathrm{b})$ or $\mathrm{T} 1 \mathrm{a} / \mathrm{b} / \mathrm{c}($ or $\mathrm{pT} 1 \mathrm{a} / \mathrm{b} / \mathrm{c})$. According to the $\mathrm{T} 1 \mathrm{a} / \mathrm{b}$ (or pT1a/b) staging, T1a (or pT1a), where tumors cells invade the lamina propria but are still located above the level of the MM and T1b (or pT1b), where tumors cells are seen invading into or beyond the MM. In $\mathrm{T} 1 \mathrm{a} / \mathrm{b} / \mathrm{c}$ (or $\mathrm{pT} 1 \mathrm{a} / \mathrm{b} / \mathrm{c}$ ) staging system, T1a (or pT1a) was defined as invasion into the stroma but not to MM, T1b (or pT1b); invasion into MM but not beyond MM, and pT1c (or pT1c); invasion beyond the MM but not to muscularis propria. Infiltration depth substaging system was defined as $\mathrm{T} 1 \mathrm{~m} / \mathrm{e}$ (or pT1 m/e). T1 m, or pT1 m (micro infiltration) was a single focus of lamina propria invasion with a maximum depth of $0.5 \mathrm{~mm}$ (within one high power field; objective $\times 40$ ). T1e or pT1/e (extensive infiltration) was defined as a larger area with invasion or multiple micro-invasive areas.

\section{Statistical analyses}

We extracted reported HRs and 95\%CIs to calculate cumulative effect size of studies which presented the association between T1(or pT1) substaging and DR and DP. Studies presented HR using multivariate Cox proportional hazard regression model were included in meta-analysis. STATA/ MPTM, version 14.2 (Stata-Corp., College Station, TX, USA) was used to perform meta-analysis. Heterogeneity between the studies included in the meta-analysis was assessed by Cochrane $Q$ test and $\mathrm{I}^{2}$ statistics. An $\mathrm{I}^{2}>50 \%$ and $p$ value $<0.05$ in Cochrane $Q$ test implied that the heterogeneity existed. With no heterogeneity among selected studies, we considered fixed effect models to calculate pooled HRs. In case of significant heterogeneity, we used random effect model. Visual inspection of funnel plot was carried out to identify publication bias in our meta-analysis.

\section{Risk of bias (RoB) assessment}

The RoB assessment of each study was done according to the Cochrane Handbook for Systematic Reviews of Interventions for including nonrandomized studies $[11,12]$. The confounding factors including treatment modality, tumor grade, carcinoma in situ (CIS), multifocality, T1 (or pT1) substaging, and tumor size were identified as the most important prognostic factors. The presence of confounders was determined by consensus. The RoB assessment for each study was performed by two independent authors and the overall RoB level was presented as "low", "intermediate", or "high" risk. 


\section{Results}

\section{Literature search process}

A total of 4999 studies were found after an initial search; 3036 records remained after exclusion of duplicates (Fig. 1). After exclusion of non-relevant studies, review articles, case reports, comments, replies, meeting abstracts, and studies in other than English, 57 studies remained. Finally, 36 and 12 studies were included for qualitative and quantitative evidence synthesis, respectively.

\section{Characteristics of the included studies}

Tables 1 and 2 summarize the studies' characteristics and patients' clinical data, respectively. Four studies were designed prospectively [13-16] and 32 studies were retrospective in design $[8,9,17-46]$. All studies were published between 1994 and 2018. In total, 6781 patients were included in 36 studies with 5964 patients who underwent T1 (or pT1) substaging and outcomes analysis. Twenty-three studies came from Europe, five from North America, six from Asia, and two from Europe/Canada region.

Nine studies included patients who had been substaged with both MM and tumor infiltration depth staging systems. Twenty-two studies included MM invasion substaging system only and five included patients substaged with tumor infiltration depth staging system only. TURBT with or without intravesical BCG or chemotherapy agents was reported as initial therapy in 6677 patients. Radical or partial cystectomy and/or radiation therapy were reported in 104 patients as initial therapeutic modality [13, 17, 24, 29, 35, 39, 45]. The prognostic value of $\mathrm{T} 1$ (or pT1) substaging on at least one oncological outcome was established in 29 studies.

\section{Meta-analysis}

\section{T1 (or pT1) MM invasion substaging and DP}

The impact of MM invasion on DP was investigated in patients with $\mathrm{T} 1$ (or pT1) bladder urothelial carcinoma. Overall seven studies with a total of 899 patients were identified and MM invasion was associated with a higher DP rate
Fig. 1 PRISMA flow chart for article selection process to analyze the prognostic value of $\mathrm{T} 1$ substaging on oncological outcomes in patients with non-muscle-invasive bladder urothelial carcinoma

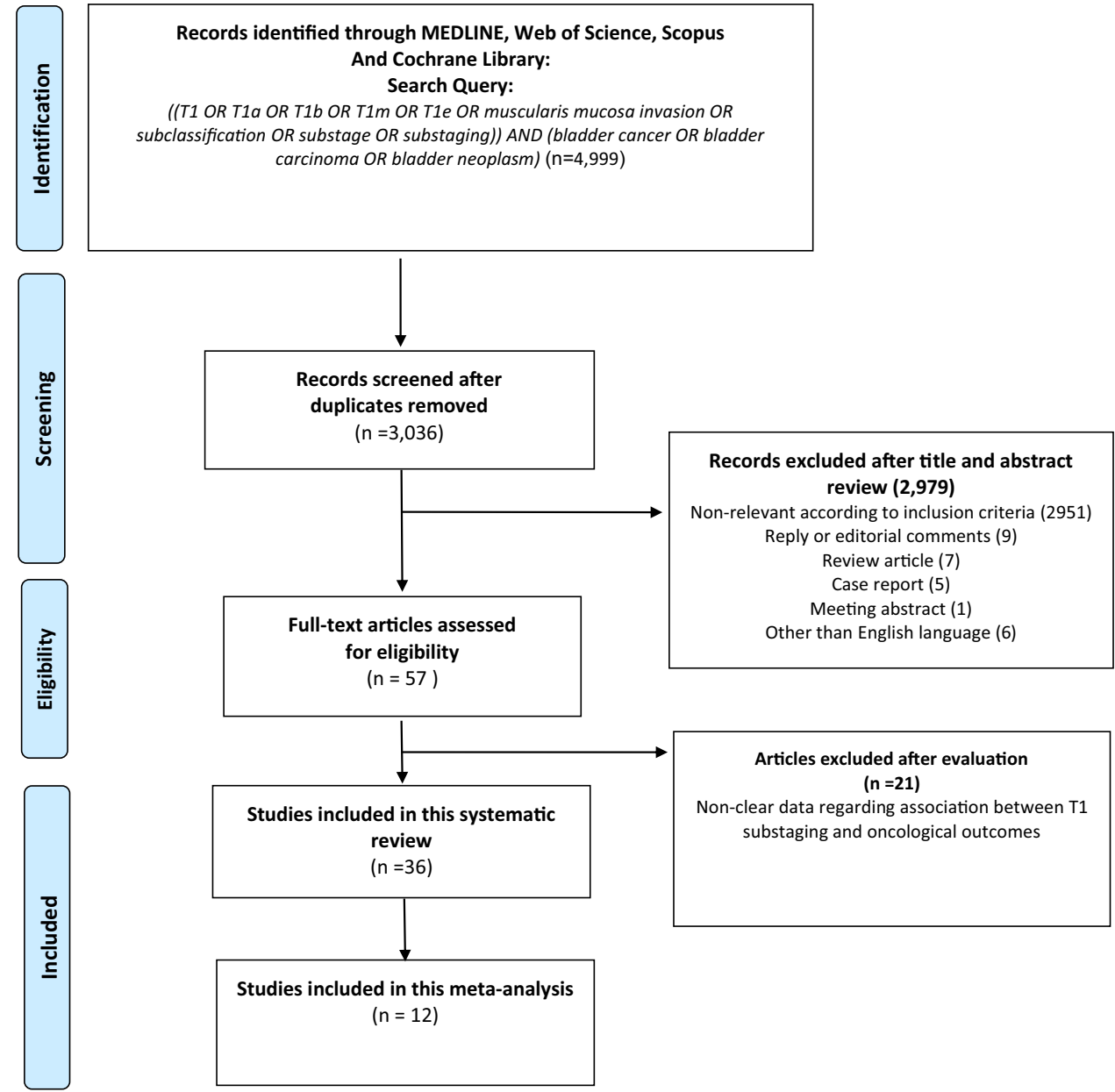


Table 1 Study characteristics of 40 studies assessing the prognostic value of T1 substaging in patients with bladder urothelial carcinoma

\begin{tabular}{|c|c|c|c|c|c|c|c|c|}
\hline Author & Year & Region & $\begin{array}{l}\text { Recruitment } \\
\text { period }\end{array}$ & Design & No.pT1 Pts & $\begin{array}{l}\text { Sub- } \\
\text { staged } \\
\text { T1 Pts }\end{array}$ & $\begin{array}{l}\text { Substaging } \\
\text { system }\end{array}$ & $\begin{array}{l}\text { Oncological end } \\
\text { point }\end{array}$ \\
\hline Hasui [23] & 1994 & Japan & 1980-1991 & Retrospective & 88 & 88 & $\begin{array}{l}\text { MM invasion } \\
\text { (T1a/T1b) }\end{array}$ & DR, DP \\
\hline Holmäng [24] & 1997 & Sweden & 1987-1988 & Retrospective & 121 & 113 & $\begin{array}{l}\text { MM invasion } \\
\text { (T1a/T1b) }\end{array}$ & DP, CSS, OS \\
\hline Smits [40] & 1998 & The Netherlands & $1987-1990$ & Retrospective & 133 & 124 & $\begin{array}{l}\text { MM invasion } \\
\text { (T1a/T1b/T1c) }\end{array}$ & DR, DP \\
\hline Cheng [22] & 1999 & USA & 1987-1992 & Retrospective & 83 & 83 & $\begin{array}{l}\text { Depth of lamina } \\
\text { propria invasion }\end{array}$ & DP \\
\hline Kondylis [26] & 2000 & USA & 1981-1997 & Retrospective & 55 & 49 & $\begin{array}{l}\text { MM invasion } \\
\text { (T1a/T1b) }\end{array}$ & DR, DP \\
\hline Shariat [39] & 2000 & USA & N/A & Retrospective & 47 & 36 & $\begin{array}{l}\text { MM invasion } \\
\text { (T1a/T1b) }\end{array}$ & DR, DP, OS \\
\hline Bernardini [17] & 2001 & France & 1973-1996 & Retrospective & 149 & 94 & $\begin{array}{l}\text { MM invasion } \\
\text { (T1a/T1b) }\end{array}$ & PFS \\
\hline Sozen [42] & 2002 & Turkey & 1983-1997 & Retrospective & 90 & 50 & $\begin{array}{l}\text { MM invasion } \\
\text { (T1a/T1b) }\end{array}$ & DR, DP \\
\hline Orsola [32] & 2005 & Spain & 1996-2001 & Retrospective & 97 & 85 & $\begin{array}{l}\text { MM invasion } \\
(\mathrm{T} 1 \mathrm{a} / \mathrm{T} 1 \mathrm{~b} / \mathrm{T} 1 \mathrm{c})\end{array}$ & RFS, PFS \\
\hline van der Aa [45] & 2005 & The Netherlands & N/A & Retrospective & 63 & 53 & $\begin{array}{l}\text { Tumor infiltration } \\
\text { depth (T1 m/ } \\
\text { T1e) }\end{array}$ & DP \\
\hline $\begin{array}{l}\text { Chaimuangraj } \\
{[20]}\end{array}$ & 2006 & Thailand & 1990-2004 & Retrospective & 192 & 192 & $\begin{array}{l}\text { Muscularis } \\
\text { mucosa inva- } \\
\text { sion }\end{array}$ & DR \\
\hline Andius [13] & 2007 & Sweden & 1987-1988 & Prospective & 121 & 121 & $\begin{array}{c}\text { MM invasion } \\
(\mathrm{T} 1 \mathrm{a} / \mathrm{T} 1 \mathrm{~b})^{\dagger}\end{array}$ & PFS, CSS \\
\hline $\begin{array}{l}\text { Mhawech-Fau- } \\
\text { ceglia [29] }\end{array}$ & 2007 & Switzerland & N/A & Retrospective & 45 & 45 & $\begin{array}{l}\text { MM invasion } \\
\text { (T1a/T1b) }\end{array}$ & DR, DP \\
\hline $\begin{array}{l}\text { Queipo-Zaragoza } \\
\text { [37] }\end{array}$ & 2007 & Spain & 1986-2003 & Retrospective & 91 & 83 & $\begin{array}{l}\text { MM invasion } \\
\text { (T1a/T1b) }\end{array}$ & DP \\
\hline Soukup [16] & 2008 & Czech Republic & 2001-2005 & Prospective & 105 & 99 & $\begin{array}{l}\text { MM invasion } \\
\text { (T1a/T1b) }\end{array}$ & DR, DP (PFS) \\
\hline Orsola [14] & 2010 & Spain & N/A & Prospective & 159 & 138 & $\begin{array}{l}\text { MM invasion } \\
(\mathrm{T} 1 \mathrm{a} / \mathrm{T} 1 \mathrm{~b})\end{array}$ & DR, DP \\
\hline Bertz [18] & 2011 & Germany & 1989-2006 & Retrospective & 309 & 309 & $\begin{array}{l}\text { MM invasion } \\
\text { (T1a/T1b), } \\
\text { Infiltration } \\
\text { depth }(\leq 1 \\
\text { HPF/> } 1 \mathrm{HPF})\end{array}$ & CSS, RFS, PFS \\
\hline Palou [34] & 2012 & Spain/Belgium & 1985-1996 & Retrospective & 146 & 93 & $\begin{array}{l}\text { MM invasion } \\
(\mathrm{T} 1 \mathrm{a} / \mathrm{T} 1 \mathrm{~b} / \mathrm{T} 1 \mathrm{c})\end{array}$ & DR, DP, CSM \\
\hline Lee [27] & 2012 & Korea & 1999-2009 & Retrospective & 183 & 183 & $\begin{array}{l}\text { MM invasion } \\
\text { (T1a/T1b/T1c) }\end{array}$ & DR, DP, CSM \\
\hline Chang [21] & 2012 & Taiwan & $1991-2005$ & Retrospective & 509 & 509 & $\begin{array}{l}\text { Muscularis } \\
\text { mucosa inva- } \\
\text { sion, Infiltra- } \\
\text { tion depth ( } 3 \\
\text { cut-off values } \\
\text { to substage } \\
\text { the } \mathrm{T} 1 \text { tumors: } \\
0.5 \mathrm{~mm} \text {, } \\
1.0 \mathrm{~mm} \text {, and } \\
1.5 \mathrm{~mm} \text { ) }\end{array}$ & $\mathrm{DR}, \mathrm{DP}, \mathrm{CSD}, \mathrm{OM}$ \\
\hline
\end{tabular}


Table 1 (continued)

\begin{tabular}{|c|c|c|c|c|c|c|c|c|}
\hline Author & Year & Region & $\begin{array}{l}\text { Recruitment } \\
\text { period }\end{array}$ & Design & No.pT1 Pts & $\begin{array}{l}\text { Sub- } \\
\text { staged } \\
\text { T1 Pts }\end{array}$ & $\begin{array}{l}\text { Substaging } \\
\text { system }\end{array}$ & $\begin{array}{l}\text { Oncological end } \\
\text { point }\end{array}$ \\
\hline van Rhijn [46] & 2012 & $\begin{array}{l}\text { The Netherlands/ } \\
\text { Canada }\end{array}$ & 1984-2006 & Retrospective & 129 & 129 & $\begin{array}{l}\text { MM invasion } \\
\text { (T1a/T1b/T1c), } \\
\text { tumor infiltra- } \\
\text { tion depth } \\
\text { (T1 m/T1e) }\end{array}$ & DR, DP \\
\hline Brimo [19] & 2013 & Canada & 2004-2012 & Retrospective & 86 & 86 & $\begin{array}{l}\text { Muscularis } \\
\text { mucosa inva- } \\
\text { sion, Maximum } \\
\text { tumor depth } \\
(\mathrm{mm})\end{array}$ & DR,DP,WFS \\
\hline Olsson [31] & 2013 & Sweden & $1992-2001$ & Retrospective & 285 & 211 & $\begin{array}{l}\text { MM invasion } \\
(\mathrm{T} 1 \mathrm{a} / \mathrm{T} 1 \mathrm{~b} / \mathrm{T} 1 \mathrm{c})\end{array}$ & DR, DP \\
\hline Nishiyama [30] & 2013 & Japan & 1995-2010 & Retrospective & 79 & 79 & $\begin{array}{l}\text { Tumor infiltration } \\
\text { depth (T1 m/ } \\
\text { T1e) }\end{array}$ & DR, DP \\
\hline Rouprêt [38] & 2013 & France & 1994-2010 & Retrospective & 612 & 587 & $\begin{array}{l}\text { MM invasion } \\
\text { (T1a/T1b) }\end{array}$ & RFS, PFS, CSS \\
\hline Soukup [41] & 2014 & Czech Republic & 2002-2009 & Retrospective & 200 & 176 & $\begin{array}{l}\text { MM invasion } \\
\text { (T1a/T1b) }\end{array}$ & $\begin{array}{l}\text { RFS, PFS, CSS, } \\
\text { OS }\end{array}$ \\
\hline $\mathrm{Hu}[25]$ & 2014 & USA & 1997-2005 & Retrospective & 39 & 23 & $\begin{array}{l}\text { Focality, Percent- } \\
\text { age of tumor } \\
\text { invasion, and } \\
\text { aggregate } \\
\text { length of inva- } \\
\text { sion }\end{array}$ & DR \\
\hline D. E. Marco [44] & 2014 & Italy & 2000-2006 & Retrospective & 40 & 40 & $\begin{array}{l}\text { MM invasion } \\
\text { (T1a/T1b/T1c), } \\
\text { tumor infiltra- } \\
\text { tion depth } \\
\text { (T1 m/T1e) }\end{array}$ & CSS, DP \\
\hline Lim [28] & 2015 & Korea & 1998-2012 & Retrospective & 177 & 141 & $\begin{array}{l}\text { MM invasion } \\
(\mathrm{T} 1 \mathrm{a} / \mathrm{T} 1 \mathrm{~b} / \mathrm{T} 1 \mathrm{c})\end{array}$ & RFS, PFS \\
\hline Orsola [15] & 2015 & Spain & N/A & Prospective & 200 & 200 & $\begin{array}{l}\text { MM invasion } \\
\text { (T1a/T1b) }\end{array}$ & DR, DP \\
\hline Patschan [36] & 2015 & Sweden & 1997-2003 & Retrospective & 167 & 152 & $\begin{array}{l}\text { MM invasion } \\
(\mathrm{T} 1 \mathrm{a} / \mathrm{T} 1 \mathrm{~b} / \mathrm{T} 1 \mathrm{c})\end{array}$ & PFS \\
\hline Patriarca [35] & 2016 & Italy & 2011-2007 & Retrospective & 450 & 314 & $\begin{array}{l}\text { MM inva- } \\
\text { sion (T1a/ } \\
\text { T1b), tumor } \\
\text { infiltration } \\
\text { depth (T1 m/ } \\
\text { T1e), ROL } \\
\text { substaging }^{\dagger}\end{array}$ & DR, DP \\
\hline Colombo [8] & 2018 & Italy & 2007-2011 & Retrospective & 502 & 250 & $\begin{array}{l}\text { MM invasion } \\
\text { (T1a/T1b/ } \\
\text { T1c), micro- } \\
\text { infiltration } \\
\text { and extended } \\
\text { infiltration of } \\
\text { LP (T1 m/T1e), } \\
\text { ROL substaging }\end{array}$ & DR, DP \\
\hline
\end{tabular}


Table 1 (continued)

\begin{tabular}{|c|c|c|c|c|c|c|c|c|}
\hline Author & Year & Region & $\begin{array}{l}\text { Recruitment } \\
\text { period }\end{array}$ & Design & No.pT1 Pts & $\begin{array}{l}\text { Sub- } \\
\text { staged } \\
\text { T1 Pts }\end{array}$ & $\begin{array}{l}\text { Substaging } \\
\text { system }\end{array}$ & $\begin{array}{l}\text { Oncological end } \\
\text { point }\end{array}$ \\
\hline $\begin{array}{l}\text { Fransen van de } \\
\text { Putte [9] }\end{array}$ & 2018 & Europe/Canada & 1982-2010 & Retrospective & 601 & 601 & $\begin{array}{l}\text { MM invasion } \\
\text { (T1a/T1b), } \\
\text { microinfil- } \\
\text { tration and } \\
\text { extended infil- } \\
\text { tration of LP } \\
\text { (T1 m/T1e) }\end{array}$ & PFS, CSS \\
\hline Otto [33] & 2018 & $\begin{array}{l}\text { Germany/The } \\
\text { Netherlands }\end{array}$ & 1989-2012 & Retrospective & 322 & 322 & $\begin{array}{l}\text { Metric T1 sub- } \\
\text { stage (tumor } \\
\text { infiltration } \\
\text { depth) }\end{array}$ & PFS, CSS, OS \\
\hline Turan [43] & 2018 & Turkey & 2009-2014 & Retrospective & 106 & 106 & $\begin{array}{l}\text { MM invasion } \\
\text { (T1a/T1b), } \\
\text { tumor infiltra- } \\
\text { tion depth } \\
\text { (T1 m/T1e) }\end{array}$ & DR, DP \\
\hline
\end{tabular}

N/A not available, $L P$ lamina propria, $M M$ muscularis mucosa, $P F S$ progression-free survival, $C S M$ cancer-specific mortality, $C S S$ cancer-specific survival, $O S$ overall survival, WFS worsening-free survival, $D R$ disease recurrence, $D P$ disease progression, $R F S$ recurrence-free survival, $O M$ overall mortality, $H P F$ high power field

${ }^{\dagger}$ ROL substaging ROL1 < 1 power field (objective $20 \times$, ocular 10×/field 22, diameter $1.1 \mathrm{~mm}$ ) of invasion, approximately corresponding to invasion of the lamina propria $1 \mathrm{~mm}$ thick or less; ROL2: $>1$ power field (objective $20 \times$ ), approximately corresponding to invasion of the lamina propria more than $1 \mathrm{~mm}$ thick, or multifocal invasion with foci cumulatively amounting to invasion of the lamina propria more than $1 \mathrm{~mm}$ thick

(pooled HR 2.61, 95\%CI: 1.61-4.23) (Fig. 2A) [16, 19, 27, $28,32,41,46]$. A statistically significant heterogeneity was found among included studies using the Chi-square and $I^{2}$ tests $\left(\mathrm{I}^{\wedge} 2=54.1 \%, p=0.042\right)$; the weights were from random effect model to analyze pooled HR. Funnel plots identified one study over the pseudo 95\%CI (Fig. 2A).

\section{T1 (or pT1) MM invasion substaging and DR}

Six studies in a total of 930 patients reported HR to present the prognostic value of MM invasion on DR in T1(or pT1) urothelial bladder carcinoma patients $[16,18,19,27,40$, 46]. The overall pooled HR was 1.23 (95\% CI: 1.01-1.49) implying a significant association between $\mathrm{MM}$ invasion and DR (Fig. 2A). The Chi-square and $I^{2}$ tests did not show any significant heterogeneity $\left(\mathrm{I}^{\wedge} 2=41.4 \%, p=0.129\right)$. Funnel plots revealed one study over the pseudo 95\% CI (Fig. 2A). Figure 2B shows the RoB table of studies included in the T1(or pT1) MM invasion substaging meta-analysis.

\section{Infiltration depth substaging and DP}

Five studies with a total of 1171 patients with T1(or pT1) bladder urothelial carcinoma reported the association of tumor infiltration depth and DP [9, 18, 30, 45, 46]. Tumor infiltration depth was associated with DP (pooled HR: 3.29, 95\%CI: 2.39-4.51) (Fig. 3A). There was no significant heterogeneity in the Cochrane $Q$ or $I^{2}$ tests $\left(\mathrm{I}^{\wedge} 2=0.0 \%\right.$, $p=0.924)$. No study was detected over the pseudo $95 \% \mathrm{CI}$ on Funnel plots (Fig. 3A).

\section{Infiltration depth substaging and DR}

The impact of infiltration depth on DR was investigated in three studies in a total of 517 patients with T1(or pT1) bladder urothelial carcinoma $[18,30,46]$. There was a significant association between infiltration depth and DR with pooled HR of 1.49 (95\%CI: 1.11-2.00) (Fig. 3A). The Chisquare and $I^{2}$ tests did not show any significant heterogeneity $\left(\mathrm{I}^{\wedge} 2=56.4 \%, p=0.101\right)$. Funnel plots identified no study over the pseudo 95\% CI (Fig. 3A). Figure 3B shows the RoB table of studies included in T1(or pT1) Infiltration depth substaging meta-analysis.

\section{Discussion}

In this systematic review and meta-analysis, we assessed the prognostic value of T1(or pT1) substaging systems on oncological outcomes in patients with T1(or pT1) bladder urothelial carcinoma. Both MM invasion and tumor infiltration depth substaging systems were strongly associated with both DR and DP after adjusting for the effects of established confounding factors (e.g., tumor grade, CIS, and multifocality). 
Table 2 Patient characteristics in 40 studies assessing the prognostic role of T1 substaging in patients with bladder urothelial carcinoma

\begin{tabular}{|c|c|c|c|}
\hline Author & Age, year (range) & $\begin{array}{l}\text { Independent correlation with oncologic } \\
\text { outcomes }\end{array}$ & Follow-up duration \\
\hline Hasui [23] & Mean: 68 (37-95) & $\mathrm{S}$ & N/A \\
\hline Holmäng [24] & Mean: 73.1 (48-97) & $\mathrm{S}$ (for DP and CSS) & $\geq 5$ years \\
\hline Smits [40] & N/A & $\mathrm{S}$ (for PFS) & Minimal follow-up: 3 years \\
\hline Cheng [22] & Mean: 71 (47-94) & $S$ & Mean: 5.2 years (range, 1 day-10.4) \\
\hline Kondylis [26] & N/A & NS & Median 71 months (range, 4-147) \\
\hline Shariat [39] & Median: 67 (30-86) & NS & Median: 79 months \\
\hline Bernardini [17] & Mean: $68.9(42-90)$ & $S$ & Mean: 64.9 months (range, 5-288) \\
\hline Sozen [42] & Median: 62 (33-84) & $\mathrm{S}$ & Mean: 68 months (range, 24-120) \\
\hline Orsola [32] & Mean: $66.4(30.3-86.8)$ & $\begin{array}{l}\text { S (in T1b/c vs T1a substaging for RFS and } \\
\text { PFS) }\end{array}$ & Mean: 53 months \\
\hline van der Aa [45] & Mean: 68 (47-90) & $\mathrm{S}$ & Median: 55 months (range, 9-228) \\
\hline Chaimuangraj [20] & Mean: 60 (43-83) & S & N/A \\
\hline Andius [13] & Median: 74 (48-98) & NS & Median: 15 years for alive cases \\
\hline Mhawech-Fauceglia [29] & Mean: 70 & $S$ (for DP) & Median: 12 months \\
\hline Queipo-Zaragoza [37] & Mean: 68.1 & $S$ & Mean: 57.8 months (range, 13-24) \\
\hline Soukup [16] & Mean: 68.43 (38-87) & $\mathrm{S}$ (for PFS) & Mean: 23.31 months \\
\hline Orsola [14] & Mean: 69 & $\mathrm{~S}$ (for DP) & Median: 20.3 months \\
\hline Bertz [18] & Median: 71.7 (38-87 years). & $\begin{array}{l}\mathrm{S} \text { (in Infiltration depth: } \leq 1 \mathrm{HPF} \text { vs }>1 \mathrm{HPF} \\
\text { for RFS and PFS) }\end{array}$ & Mean: 49 months (range, 5-172) \\
\hline Palou [34] & Mean: $64.9(25-81)$ & NS & Median: 8.7 years \\
\hline Lee [27] & Mean: 63.5 years $(27-93)$ & $\mathrm{S}$ (for DP and CSM) & Mean: 43.5 months (range, $12-146$ ) \\
\hline Chang [21] & Mean: 71 (23-92) & $\begin{array}{l}\text { S (MM invasion: S for DP, CSM, and OM) } \\
\text { (depth of high-grade tumor: S for DR, DP, } \\
\text { CSM, OM) }\end{array}$ & $\begin{array}{l}\text { Mean: } 88 \text { months (range, 1-240) for } \\
\text { patients who were alive } \\
\text { Mean: } 39 \text { months (range, 1-193) for } \\
\text { patients who died }\end{array}$ \\
\hline van Rhijn [46] & Mean: 68.8 & $\mathrm{~S}$ (in $\mathrm{T} 1 \mathrm{~m} / \mathrm{T} 1 \mathrm{e}$ for $\mathrm{DP}$ ) & Median: 6.5 years \\
\hline Brimo [19] & Mean: 71 & $\mathrm{~S}$ & Mean: 29 months \\
\hline Olsson [31] & Median: 74 & $\begin{array}{l}\mathrm{S} \text { (in T1b/c vs T1a substaging for DP in } \\
\text { patients older than } 73 \text { years) }\end{array}$ & Median: 60 months \\
\hline Nishiyama [30] & Mean: 68.5 & $S$ (for DR) & Mean: 74.0 months \\
\hline Rouprêt [38] & Median: 70 & $S$ & Mean: 44 months (range, 6-161) \\
\hline Soukup [41] & Median: 68.83 (17.55-86.94) & $\mathrm{S}$ (for PFS, CSS, OS) & Median: 3.13 years $(0.1-10.5)$ \\
\hline $\mathrm{Hu}[25]$ & Mean: 70 years $(56-94)$ & $\mathrm{S}$ (in aggregate length of invasion; $>0.5 \mathrm{~cm}$ ) & N/A \\
\hline D. E. Marco [44] & Mean: 69.9 & NS & Median: 9.5 years \\
\hline $\operatorname{Lim}[28]$ & Mean: 68.9 (20-93) & $\mathrm{S}$ (for PFS) & Mean: 73.3 months (range, 3.9-187.9) \\
\hline Orsola [15] & Median: 71 & $\mathrm{~S}$ (for DP) & Median: 71 months (range: 5-107) \\
\hline Patschan [36] & Median: 74 & NS & ( 3 years follow-up in analysis) \\
\hline Patriarca [35] & Mean: $71.3(64-79)$ & S (in ROL1 VS ROL 2 substaging for DP) & Mean: 46 months \\
\hline Colombo $[8]$ & Mean: $70(64-77)$ & $\mathrm{S}$ (for DP in ROL2 vs ROL1 substaging) & Median: of 60 months \\
\hline Fransen van de Putte [9] & Median: 71 & $\begin{array}{l}\mathrm{S} \text { (for PFS and CSS in T1e vs T1 m substag- } \\
\text { ing) }\end{array}$ & Median: 5.9 years \\
\hline Otto [33] & Median: 72 & NS & Median: 42 months \\
\hline Turan [43] & Mean: 67.9 & $\mathrm{~S}$ (in $\mathrm{T} 1 \mathrm{a} / \mathrm{b}$ substaging for $\mathrm{DR}$ ) & Mean: 54 months \\
\hline
\end{tabular}

N/A not available, $S$ significant, $N S$ non-significant, $M M$ muscularis mucosa $P F S$ progression-free survival, $C S M$ cancer-specific mortality, CSS cancer-specific survival, $O S$ overall survival, $O M$ overall mortality, $D R$ disease recurrence, $D P$ disease progression, $R F S$ recurrence-free survival, $H P F$ high power field

${ }^{\dagger} S$ statistical significance $p$ value $<0.05$ 


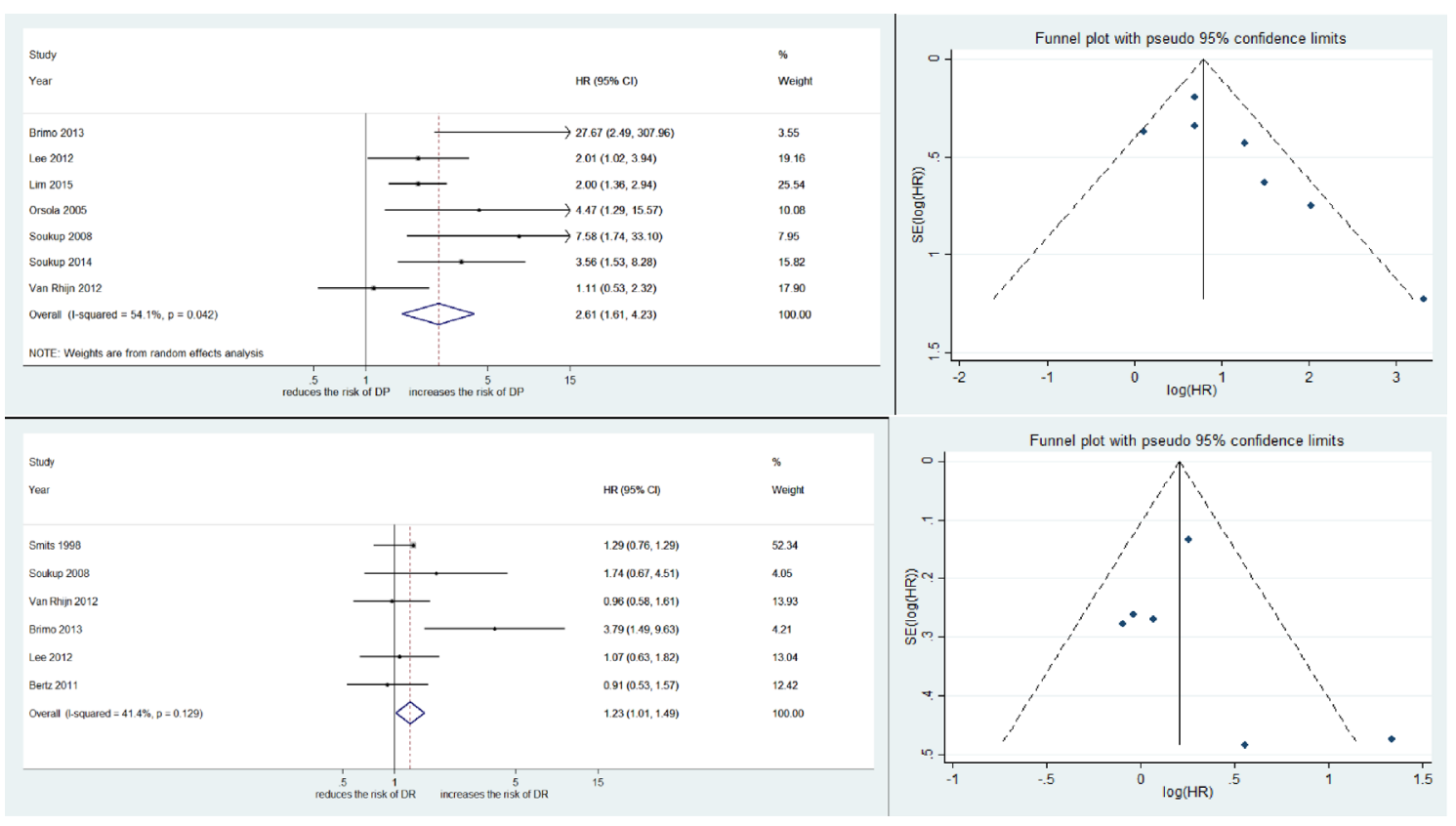

(A)

\begin{tabular}{|c|c|c|c|c|c|c|c|c|c|c|c|c|}
\hline Author, year & a & b & $\mathrm{c}$ & d & e & $\mathrm{f}$ & g & h & $\mathrm{i}$ & j & k & 1 \\
\hline Smits 1998 & - & - & • & ? & ๑ & $?$ & (†) & () & (†) & (†) & e & 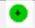 \\
\hline Orsola 2005 & • & • & • & - & • & $\odot$ & - & • & - & • & • & • \\
\hline Soukup 2008 & e & $\bullet$ & • & - & • & $\odot$ & $\bullet$ & $\odot$ & $\odot$ & $\odot$ & $\bullet$ & $\odot$ \\
\hline Lee 2012 & • & • & • & - & • & - & - & - & - & - & • & - \\
\hline van Rhijn 2012 & • & $\bullet$ & $\bullet$ & ? & $\odot$ & • & $?$ & • & • & • & • & $\odot$ \\
\hline Brimo 2013 & ○ & $\bullet$ & ? & - & - & • & - & • & - & $\bullet$ & $?$ & - \\
\hline Soukup 2014 & $\bullet$ & • & • & ? & • & - & • & $\odot$ & - & • & • & $\odot$ \\
\hline Lim 2015 & ○ & • & $\bullet$ & P & $\odot$ & • & $?$ & - & • & • & $\bullet$ & - \\
\hline
\end{tabular}

(B)

Fig. 2 A Forest plots and funnel plot of studies investigating the association of $\mathrm{T} 1 \mathrm{a} / \mathrm{b} / \mathrm{c}$ substaging system with disease progression (DP) and disease recurrence (DR) outcomes. B T1a/b/c substaging system RoB table, a Random sequence generation (selection bias). b Allocation concealment (selection bias). c Blinding of participants and personnel (Performance bias.). d Blinding of outcome assessment (detection bias). e Incomplete outcome data (attrition bias). f Selec-

The most widely used prognostic tools, taking into account tumor grade and stage, prior recurrences, tumor size, multifocality, and the presence of CIS, are still suboptimal to predict DR and DP. Moreover, the lack of effective bladder cancer information among general public may be as an important factor affecting patients' outcomes and online information and social media could be effective to improve quality of patient's care and disease management in patients with bladder cancer [47].

We and others have shown that the current prognostic and risk stratification tools are too inaccurate to guide clinical decision making safely $[1,48,49]$. In this review and metaanalysis, we confirm that tumor invasion into MM and tumor infiltration depth of more than $0.5 \mathrm{~mm}$ are strong predictors tive reporting (reporting bias); and adjustment for the effects of the following confounders. $\mathbf{g}$ Treatment modality. h Tumor grade. i CIS. j Multifocality. k T1 m/e substaging. 1 Tumor size. Green circles: low risk of bias and confounding, red circles: high risk of bias and confounding, yellow circles: unclear risk of bias and confounding. $C I$ confidence interval, $H R$ hazard ratio

of disease recurrence and progression and could be used to distinguish high risk patients for recurrence and progression who might benefit from standard adjuvant therapy (e.g., intravesical immunotherapy or chemotherapy). From these who are most likely to benefit from intensification of care such as early radical cystectomy.

In patients with NMIBC, the probability of disease progression can be as high as $45 \%$ at five years [50]. Although it has been suggested that MM substaging might be helpful to identify high risk patients who are likely to suffer from disease progression despite adequate intravesical therapy, available data quality has not been of high quality and prognostic tools have not included this valuable parameter [38, 51]. Martin-Doyle et al. evaluated 


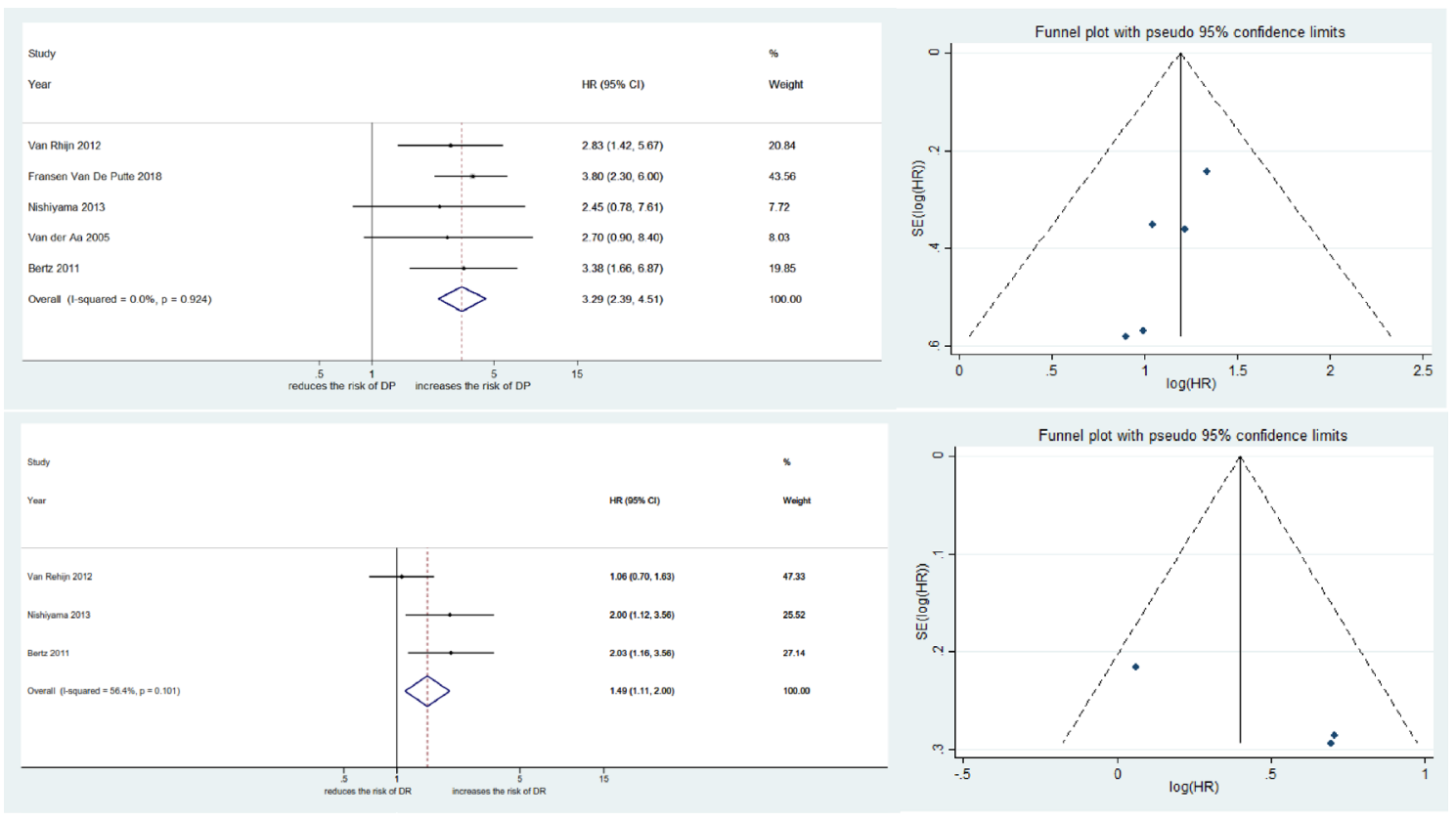

(A)

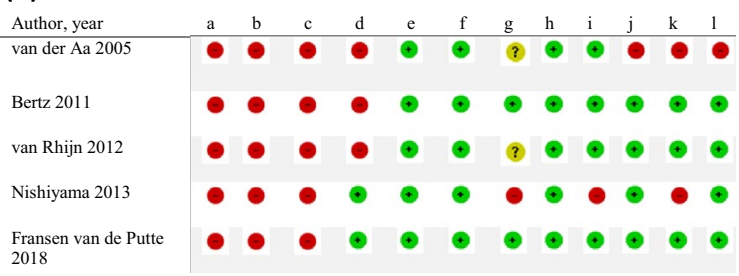

(B)

Fig. 3 A Forest plots and funnel plot of studies investigating the association of $\mathrm{T} 1 \mathrm{~m} / \mathrm{e}$ substaging system with disease progression (DP) and disease recurrence (DR) outcomes. B T1 m/e substaging system RoB table, a Random sequence generation (selection bias). b Allocation concealment (selection bias). c Blinding of participants and personnel (Performance bias.). d Blinding of outcome assessment (detection bias). e Incomplete outcome data (attrition bias). f Selec-

the prognosticators to improve selection criteria for early cystectomy in patients with high-grade $\mathrm{T} 1$ bladder cancer in a meta-analysis. The authors reported $\mathrm{T} 1 \mathrm{a} / \mathrm{b}$ substaging system as a valuable prognosticator of oncological outcomes comparable with our study with pooled HR of 1.81 (95\%CI: $0.88-3.73$ ) for DR and pooled HR of 3.55 (95\% CI: 1.92-6.56) for DP in 420 and 785 patients with high-grade T1 bladder cancer, respectively [51]. We confirmed that both MM invasion and tumor infiltration depth are strong predictors of disease progression after controlling for the effect of standard prognosticators. Indeed, patients harboring $\mathrm{T} 1 \mathrm{~b} / \mathrm{c}$ or $\mathrm{T} 1 \mathrm{e}$ in substaging system may benefit from early radical cystectomy as their tumor carries the biologic and clinical behavior of muscle-invasive bladder cancer [51]. In patients considered candidates for radical cystectomy, pretreatment imaging modalities including magnetic resonance imaging and positron emission tive reporting (reporting bias); and adjustment for the effects of the following confounders. $\mathbf{g}$ treatment modality. $\mathbf{h}$ tumor grade. i CIS. j Multifocality. k T1a/b/c substaging. 1 Tumor size. Green circles: low risk of bias and confounding, red circles: high risk of bias and confounding, yellow circles: unclear risk of bias and confounding. $C I$ confidence interval, $H R$ hazard ratio

topography/computed tomography (CT) provide higher sensitivity and similar specificity compared to CT for detection of positive lymph nodes that might have a significant impact on clinical decision-making process [52].

A consensus among pathologists is urgent to propose $\mathrm{T} 1$ (or pT1) substaging systems as a prognosticator in TNM classification system and guidelines. MM is identified in $12-83 \%$ of bladder biopsy specimen [53, 54]. Therefore, some studies proposed identification of large vessels of the vascular plexus as an alternative tumor extension marker in specimens without obvious MM [43, 46]. Moreover, although a cut-off point of $5 \mathrm{~mm}$ has been proposed in several studies to define tumor infiltration depth, other studies have utilized other definitions $[8,35]$. These discrepancies between definitions may lead to low reproducibility and questionable validity. Standardization and prospective assessment in controlled studies is necessary. 
According to our study, although substaging of T1(or pT1) disease is somewhat controversial and difficult to implement in all cases; the main advantage of this scoring system is to identify the high risk T1 bladder cancer patients who might benefit from more rigorous follow-up and ideally from more aggressive treatments which are appropriate for invasive bladder carcinoma.

This study is not without limitations. The majority of included studies in this systemic review were retrospective in design precluding robust conclusions about the prognostic value of T1(or pT1) substaging systems. Moreover, the heterogeneity of substaging systems was found in MM invasion and tumor infiltration depth systems as well as the outcomes assessed in the studies makes clear conclusions difficult. Indeed, further studies are needed to assess the prognostic value of $\mathrm{T} 1$ (or pT1) substaging systems in patient counselling and risk-based selection of the personalized therapeutic modality.

\section{Conclusion}

We found that T1(or pT1) substaging systems are strong predictors of oncological outcomes (DR, DR). Although T1(or pT1) substaging systems are promising and can be used as an aid in determining the most appropriate treatment modality and intensity of follow-up, optimal T1(or pT1) substaging system definition remains to be elucidated in future well-designed prospective studies.

Acknowledgements Open access funding provided by Medical University of Vienna.

Author contribution Protocol/project development: S.F Shariat, M Kardoust Parizi, H Fajkovic1. Data extraction or management: M Kardoust Parizi, V Seebacher, P.V Glybochko. Data analysis: M Kardoust Parizi, D Enikeev. Manuscript writing: M Kardoust Parizi, P.L Chłosta, F Janisch. Manuscript editing and supervision: S.F Shariat, M Kardoust Parizi.

\section{Compliance with ethical standards}

Conflict of interest The authors declare that they have no conflict of interest.

Ethical approval This article does not contain any studies using human participants or animals.

Informed consent This article does not contain any studies using human participants.

Open Access This article is distributed under the terms of the Creative Commons Attribution 4.0 International License (http://creativeco mmons.org/licenses/by/4.0/), which permits unrestricted use, distribution, and reproduction in any medium, provided you give appropriate credit to the original author(s) and the source, provide a link to the Creative Commons license, and indicate if changes were made.

\section{References}

1. Soukup V, Capoun O, Cohen D, Hernandez V, Burger M, Comperat E, Gontero P, Lam T, Mostafid AH, Palou J, van Rhijn BWG, Roupret M, Shariat SF, Sylvester R, Yuan Y, Zigeuner R, Babjuk M (2018) Risk stratification tools and prognostic models in nonmuscle-invasive bladder cancer: a critical assessment from the european association of urology non-muscle-invasive bladder cancer guidelines panel. Eur Urol Focus. https://doi.org/10.1016/j. euf.2018.11.005

2. Kluth LA, Xylinas E, Crivelli JJ, Passoni N, Comploj E, Pycha A, Chrystal J, Sun M, Karakiewicz PI, Gontero P, Lotan Y, Chun FK, Fisch M, Scherr DS, Shariat SF (2013) Obesity is associated with worse outcomes in patients with $\mathrm{T} 1$ high grade urothelial carcinoma of the bladder. J Urol 190(2):480-486. https://doi. org/10.1016/j.juro.2013.01.089

3. Fritsche HM, Burger M, Svatek RS, Jeldres C, Karakiewicz PI, Novara G, Skinner E, Denzinger S, Fradet Y, Isbarn H, Bastian PJ, Volkmer BG, Montorsi F, Kassouf W, Tilki D, Otto W, Capitanio U, Izawa JI, Ficarra V, Lerner S, Sagalowsky AI, Schoenberg M, Kamat A, Dinney CP, Lotan Y, Shariat SF (2010) Characteristics and outcomes of patients with clinical T1 grade 3 urothelial carcinoma treated with radical cystectomy: results from an international cohort. Eur Urol 57(2):300-309. https://doi.org/10.1016/j. eururo.2009.09.024

4. Tilki D, Shariat SF, Lotan Y, Rink M, Karakiewicz PI, Schoenberg MP, Lerner SP, Sonpavde G, Sagalowsky AI, Gupta A (2013) Lymphovascular invasion is independently associated with bladder cancer recurrence and survival in patients with final stage $\mathrm{T} 1$ disease and negative lymph nodes after radical cystectomy. BJU Intern 111(8):1215-1221. https://doi.org/10.1111/j.1464410X.2012.11455.x

5. Mari A, Kimura S, Foerster B, Abufaraj M, D'Andrea D, Hassler M, Minervini A, Roupret M, Babjuk M, Shariat SF (2019) A systematic review and meta-analysis of the impact of lymphovascular invasion in bladder cancer transurethral resection specimens. BJU Intern 123(1):11-21. https://doi.org/10.1111/bju.14417

6. Abufaraj M, Foerster B, Schernhammer E, Moschini M, Kimura S, Hassler MR, Preston MA, Karakiewicz PI, Remzi M, Shariat SF (2019) Micropapillary urothelial carcinoma of the bladder: a systematic review and meta-analysis of disease characteristics and treatment outcomes. Eur Urol 75(4):649-658. https://doi. org/10.1016/j.eururo.2018.11.052

7. Da D, Abufaraj M, Susani M, Ristl R, Foerster B, Kimura S, Mari A, Soria F, Briganti A, Karakiewicz PI, Gust KM, Roupret M, Shariat SF (2018) Accurate prediction of progression to muscleinvasive disease in patients with pT1G3 bladder cancer: a clinical decision-making tool. Urol Oncol 36(5):239.e231-239.e237. https ://doi.org/10.1016/j.urolonc.2018.01.018

8. Colombo R, Hurle R, Moschini M, Freschi M, Colombo P, Colecchia M, Ferrari L, Luciano R, Conti G, Magnani T, Capogrosso P, Conti A, Pasini L, Burgio G, Guazzoni G, Patriarca C (2018) Feasibility and clinical roles of different substaging systems at first and second transurethral resection in patients with t 1 highgrade bladder cancer. Eur Urol Focus 4(1):87-93. https://doi. org/10.1016/j.euf.2016.06.004

9. Fransen van de Putte EE, Otto W, Hartmann A, Bertz S, Mayr R, Brundl J, Breyer J, Manach Q, Comperat EM, Boormans JL, Bosschieter J, Jewett MAS, Stoehr R, van Leenders G, Nieuwenhuijzen JA, Zlotta AR, Hendricksen K, Roupret M, Burger M, van der Kwast TH, van Rhijn BWG (2018) Metric substage according to micro and extensive lamina propria invasion improves prognostics in T1 bladder cancer. Urol Oncol 36(8):e7-e13. https://doi. org/10.1016/j.urolonc.2018.05.007 
10. Liberati A, Altman DG, Tetzlaff J, Mulrow C, Gøtzsche PC, Ioannidis JPA, Clarke M, Devereaux PJ, Kleijnen J, Moher D (2009) The PRISMA statement for reporting systematic reviews and meta-analyses of studies that evaluate health care interventions: explanation and elaboration. J Clin Epidemiol 62(10):e1-e34. https://doi.org/10.1016/j.jclinepi.2009.06.006

11. Deeks JJ, Dinnes J, D'Amico R, Sowden AJ, Sakarovitch C, Song F, Petticrew M, Altman DG (2003) Evaluating non-randomised intervention studies. Health Technology Assessment, Winchester, pp 1-173

12. Higgins JPT, Green $S$ (editors). Cochrane handbook for systematic reviews of interventions version 5.1.0 [updated March 2011]. The cochrane collaboration (2011). Available from http://handbook. cochrane.org

13. Andius P, Johansson SL, Holmang S (2007) Prognostic factors in stage $\mathrm{T} 1$ bladder cancer: tumor pattern (solid or papillary) and vascular invasion more important than depth of invasion. Urology 70(4):758-762. https://doi.org/10.1016/j.urology.2007.06.638

14. Orsola A, Cecchini L, Raventos CX, Trilla E, Planas J, Landolfi S, de Torres I, Morote J (2010) Risk factors for positive findings in patients with high-grade $\mathrm{T} 1$ bladder cancer treated with transurethral resection of bladder tumour (TUR) and bacille Calmette-Guerin therapy and the decision for a repeat TUR. BJU Int 105(2):202-207. https://doi.org/10.1111/j.1464-410X.2009.08694 . $\mathrm{x}$

15. Orsola A, Werner L, de Torres I, Martin-Doyle W, Raventos CX, Lozano F, Mullane SA, Leow JJ, Barletta JA, Bellmunt J, Morote J (2015) Reexamining treatment of high-grade T1 bladder cancer according to depth of lamina propria invasion: a prospective trial of 200 patients. Br J Cancer 112(3):468-474. https://doi. org/10.1038/bjc.2014.633

16. Soukup V, Babjuk M, Duskova J, Pesl M, Szakaczova M, Zamecnik L, Dvoracek J (2008) Does the expression of fascin-1 and tumor subclassification help to assess the risk of recurrence and progression in $\mathrm{t} 1$ urothelial urinary bladder carcinoma? Urol Int 80(4):413-418. https://doi.org/10.1159/000132700

17. Bernardini S, Billerey C, Martin M, Adessi GL, Wallerand H, Bittard $\mathrm{H}$ (2001) The predictive value of muscularis mucosae invasion and p53 over expression on progression of stage $\mathrm{T} 1$ bladder carcinoma. J Urol 165(1):42-46. https://doi.org/10.1097/00005 392-200101000-00011

18. Bertz S, Denzinger S, Otto W, Wieland WF, Stoehr R, Hofstaedter F, Hartmann A (2011) Substaging by estimating the size of invasive tumour can improve risk stratification in pT1 urothelial bladder cancer-evaluation of a large hospital-based single-centre series. Histopathology 59(4):722-732. https://doi.org/10.111 $1 / j .1365-2559.2011 .03989 . x$

19. Brimo F, Wu C, Zeizafoun N, Tanguay S, Aprikian A, Mansure JJ, Kassouf W (2013) Prognostic factors in T1 bladder urothelial carcinoma: the value of recording millimetric depth of invasion, diameter of invasive carcinoma, and muscularis mucosa invasion. Hum Pathol 44(1):95-102. https://doi.org/10.1016/j.humpa th.2012.04.020

20. Chaimuangraj S, Dissaranan C, Leenanupunth C, Prathombutr P, Chalermsanyakorn P (2006) Significance of muscularis mucosae in metastasis involvement of urinary bladder transitional cell carcinoma. J Med Assoc Thai 89(9):1447-1453

21. Chang WC, Chang YH, Pan CC (2012) Prognostic significance in substaging of 11 urinary bladder urothelial carcinoma on transurethral resection. Am J Surg Pathol 36(3):454-461. https://doi. org/10.1097/PAS.0b013e31823dafd3

22. Cheng L, Neumann RM, Weaver AL, Spotts BE, Bostwick DG (1999) Predicting cancer progression in patients with stage T1 bladder carcinoma. J Clin Oncol 17(10):3182-3187. https://doi. org/10.1200/jco.1999.17.10.3182
23. Hasui Y, Osada Y, Kitada S, Nishi S (1994) Significance of invasion to the muscularis mucosae on the progression of superficial bladder cancer. Urology 43(6):782-786

24. Holmäng S, Hedelin H, Anderström C, Holmberg E, Johansson SL (1997) The importance of the depth of invasion in stage T1 bladder carcinoma: a prospective cohort study. J Urol 157(3):800-804. https://doi.org/10.1016/S0022-5347(01)65044-4

25. Hu Z, Mudaliar K, Quek ML, Paner GP, Barkan GA (2014) Measuring the dimension of invasive component in pT1 urothelial carcinoma in transurethral resection specimens can predict time to recurrence. Ann Diagn Pathol 18(2):49-52. https://doi. org/10.1016/j.anndiagpath.2013.11.002

26. Kondylis FI, Demirci S, Ladaga L, Kolm P, Schellhammer PF (2000) Outcomes after intravesical bacillus Calmette-Guerin are not affected by substaging of high grade $\mathrm{T} 1$ transitional cell carcinoma. J Urol 163(4):1120-1123

27. Lee JY, Joo HJ, Cho DS, Kim SI, Ahn HS, Kim SJ (2012) Prognostic significance of substaging according to the depth of lamina propria invasion in primary $\mathrm{T} 1$ transitional cell carcinoma of the bladder. Korean J Urol 53(5):317-323. https://doi. org/10.4111/kju.2012.53.5.317

28. Lim SD, Cho YM, Choi GS, Park HK, Paick SH, Kim WY, Kim SN, Yoon G (2015) Clinical significance of substaging and HER2 expression in papillary nonmuscle invasive urothelial cancers of the urinary bladder. J Korean Med Sci 30(8):10681077. https://doi.org/10.3346/jkms.2015.30.8.1068

29. Mhawech-Fauceglia P, Fischer G, Alvarez V Jr, Ahmed A, Herrmann FR (2007) Predicting outcome in minimally invasive (T1a and $\mathrm{T} 1 \mathrm{~b})$ urothelial bladder carcinoma using a panel of biomarkers: a high throughput tissue microarray analysis. BJU Int 100(5):1182-1187. https://doi.org/10.1111/j.1464410X.2007.07090.x

30. Nishiyama N, Kitamura H, Maeda T, Takahashi S, Masumori N, Hasegawa T, Tsukamoto T (2013) Clinicopathological analysis of patients with non-muscle-invasive bladder cancer: prognostic value and clinical reliability of the 2004 WHO classification system. Jpn J Clin Oncol 43(11):1124-1131. https://doi. org/10.1093/jjco/hyt120

31. Olsson H, Hultman P, Rosell J, Jahnson S (2013) Populationbased study on prognostic factors for recurrence and progression in primary stage T1 bladder tumours. Scand J Urol 47(3):188-195. https://doi.org/10.3109/00365599.2012.719539

32. Orsola A, Trias I, Raventos CX, Espanol I, Cecchini L, Bucar S, Salinas D, Orsola I (2005) Initial high-grade T1 urothelial cell carcinoma: feasibility and prognostic significance of lamina propria invasion microstaging $(\mathrm{T} 1 \mathrm{a} / \mathrm{b} / \mathrm{c})$ in BCG-treated and BCG-non-treated patients. Eur Urol 48(2):231-238. https:// doi.org/10.1016/j.eururo.2005.04.013

33. Otto W, van Rhijn BW, Breyer J, Bertz S, Eckstein M, Mayr R, Lausenmeyer EM, Denzinger S, Burger M, Hartmann A (2018) Infiltrative lamina propria invasion pattern as an independent predictor for cancer-specific and overall survival of instillation treatment-naive stage $\mathrm{T} 1 \mathrm{high}$-grade urothelial bladder cancer. Int J Urol 25(5):442-449. https://doi.org/10.1111/iju.13532

34. Palou J, Sylvester RJ, Faba OR, Parada R, Pena JA, Algaba F, Villavicencio H (2012) Female gender and carcinoma in situ in the prostatic urethra are prognostic factors for recurrence, progression, and disease-specific mortality in T1G3 bladder cancer patients treated with bacillus Calmette-Guerin. Eur Urol 62(1):118-125. https://doi.org/10.1016/j.eururo.2011.10.029

35. Patriarca C, Hurle R, Moschini M, Freschi M, Colombo P, Colecchia M, Ferrari L, Guazzoni G, Conti A, Conti G, Luciano R, Magnani T, Colombo R (2016) Usefulness of pT1 substaging in papillary urothelial bladder carcinoma. Diag Pathol 11:6. https://doi.org/10.1186/s13000-016-0466-6 
36. Patschan O, Sjodahl G, Chebil G, Lovgren K, Lauss M, Gudjonsson S, Kollberg P, Eriksson P, Aine M, Mansson W, Ferno M, Liedberg F, Hoglund M (2015) A molecular pathologic framework for risk stratification of stage T1 urothelial carcinoma. Eur Urol 68(5):824-832. https://doi.org/10.1016/j.eurur o.2015.02.021

37. Queipo-Zaragoza JA, Ruiz-Cerda JL, Vera-Donoso CD, VeraSempere F, Budia-Alba A, Jimenez-Cruz JF (2007) Prognostic value of p53, Ki-67, microstaging and microvessel density in pT1G3 bladder tumors: creation of risk groups for progression. Scand J Urol Nephrol 41(4):283-289. https://doi. org/10.1080/00365590601183055

38. Rouprêt M, Seisen T, Compérat E, Larré S, Mazerolles C, Gobet F, Fetissof F, Fromont G, Safsaf A, D'Arcier BF, Celhay O, Validire P, Rozet F, Irani J, Soulié M, Pfister C (2013) Prognostic interest in discriminating muscularis mucosa invasion (T1a vs T1b) in nonmuscle invasive bladder carcinoma: french national multicenter study with central pathology review. J Urol 189(6):2069-2076. https://doi.org/10.1016/j.juro.2012.11.120

39. Shariat SF, Weizer AZ, Green A, Laucirica R, Frolov A, Wheeler TM, Lerner SP (2000) Prognostic value of P53 nuclear accumulation and histopathologic features in $\mathrm{T} 1$ transitional cell carcinoma of the urinary bladder. Urology 56(5):735-740

40. Smits G, Schaafsma E, Kiemeney L, Caris C, Debruyne F, Witjes JA (1998) Microstaging of pT1 transitional cell carcinoma of the bladder: identification of subgroups with distinct risks of progression. Urology 52(6):1009-1013

41. Soukup V, Duskova J, Pesl M, Capoun O, Feherova Z, Zamecnik L, Hanus T, Babjuk M (2014) The prognostic value of T1 bladder cancer substaging: a single institution retrospective study. Urol Int 92(2):150-156. https://doi.org/10.1159/000355358

42. Sozen S, Akbal C, Sokmensuer C, Ekici S, Ozen H (2002) Microstaging of pT1 transitional cell carcinoma of the bladder. Does it really differentiate two populations with different prognoses? (pT1 subcategory). Urol Int 69(3):200-206. https://doi. org/10.1159/000063941

43. Turan T, Efiloglu O, Gunaydin B, Ozkanli S, Nikerel E, Atis G, Caskurlu T, Yildirim A (2018) Comparative differences between $\mathrm{T} 1 \mathrm{a} / \mathrm{b}$ and $\mathrm{T} 1 \mathrm{e} / \mathrm{m}$ as substages in $\mathrm{T} 1$ urothelial carcinoma of the bladder. Int Braz J Urol 44(2):267-272. https://doi.org/10.1590/ s1677-5538.ibju.2017.0424

44. Dem V, Cerruto MA, D'Elia C, Brunelli M, Otte O, Minja A, Luchini C, Novella G, Cavalleri S, Martignoni G, Artibani W (2014) Prognostic role of substaging in T1G3 transitional cell carcinoma of the urinary bladder. Mol Clin Oncol 2(4):575-580. https://doi.org/10.3892/mco.2014.290

45. van der Aa MN, van Leenders GJ, Steyerberg EW, van Rhijn BW, Jobsis AC, Zwarthoff EC, van der Kwast TH (2005) A new system for substaging pT1 papillary bladder cancer: a prognostic evaluation. Hum Pathol 36(9):981-986. https://doi.org/10.1016/j.humpa th.2005.06.017
46. van Rhijn BW, van der Kwast TH, Alkhateeb SS, Fleshner NE, van Leenders GJ, Bostrom PJ, van der Aa MN, Kakiashvili DM, Bangma CH, Jewett MA, Zlotta AR (2012) A new and highly prognostic system to discern T1 bladder cancer substage. Eur Urol 61(2):378-384. https://doi.org/10.1016/j.eururo.2011.10.026

47. Tariq A, Khan SR, Vela I, Williams ED (2019) Assessment of the use of the Internet and social media among people with bladder cancer and their carers, and the quality of available patientcentric online resources: a systematic review. BJU Int 123(Suppl 5):10-18. https://doi.org/10.1111/bju.14720

48. Rieken M, Shariat SF, Kluth L, Crivelli JJ, Abufaraj M, Foerster B, Mari A, Ilijazi D, Karakiewicz PI, Babjuk M, Gonen M, Xylinas E (2018) Comparison of the EORTC tables and the EAU categories for risk stratification of patients with nonmuscle-invasive bladder cancer. Urol Oncol 36(1):8.e17-18.e24. https://doi. org/10.1016/j.urolonc.2017.08.027

49. Xylinas E, Kent M, Kluth L, Pycha A, Comploj E, Svatek RS, Lotan Y, Trinh QD, Karakiewicz PI, Holmang S, Scherr DS, Zerbib M, Vickers AJ, Shariat SF (2013) Accuracy of the EORTC risk tables and of the CUETO scoring model to predict outcomes in non-muscle-invasive urothelial carcinoma of the bladder. $\mathrm{Br} \mathrm{J}$ Cancer 109(6):1460-1466. https://doi.org/10.1038/bjc.2013.372

50. Sylvester RJ, van der Meijden AP, Oosterlinck W, Witjes JA, Bouffioux C, Denis L, Newling DW, Kurth K (2006) Predicting recurrence and progression in individual patients with stage Ta $\mathrm{T} 1$ bladder cancer using EORTC risk tables: a combined analysis of 2596 patients from seven EORTC trials. Eur Urol 49(3):466-477. https://doi.org/10.1016/j.eururo.2005.12.031

51. Martin-Doyle W, Leow JJ, Orsola A, Chang SL, Bellmunt J (2015) Improving selection criteria for early cystectomy in high-grade $\mathrm{t} 1$ bladder cancer: a meta-analysis of 15,215 patients. J Clin Oncol 33(6):643-650. https://doi.org/10.1200/jco.2014.57.6967

52. Crozier J, Papa N, Perera M, Ngo B, Bolton D, Sengupta S, Lawrentschuk N (2019) Comparative sensitivity and specificity of imaging modalities in staging bladder cancer prior to radical cystectomy: a systematic review and meta-analysis. World J Urol 37(4):667-690. https://doi.org/10.1007/s00345-018-2439-8

53. Cottrell L, Nairn ER, Hair M (2007) Consistency of microstaging pT1 bladder transitional cell carcinoma. J Clin Pathol 60(6):735736. https://doi.org/10.1136/jcp.2006.045708

54. Segal R, Yafi FA, Brimo F, Tanguay S, Aprikian A, Kassouf W (2012) Prognostic factors and outcome in patients with T1 highgrade bladder cancer: can we identify patients for early cystectomy? BJU Int 109(7):1026-1030. https://doi.org/10.1111/j.1464410X.2011.10462.x

Publisher's Note Springer Nature remains neutral with regard to jurisdictional claims in published maps and institutional affiliations. 


\section{Affiliations}

\section{Mehdi Kardoust Parizi ${ }^{1,2} \cdot$ Dmitry Enikeev $^{3}$. Petr V. Glybochko ${ }^{3}$. Veronika Seebacher ${ }^{4}$ Florian Janisch $^{1,5}$. Harun Fajkovic ${ }^{1}$ - Piotr L. Chłosta ${ }^{6}$. Shahrokh F. Shariat ${ }^{1,3,7,8}$}

1 Department of Urology and Comprehensive Cancer Center, Vienna General Hospital, Medical University of Vienna, Währinger Gürtel 18-20, 1090 Vienna, Austria

2 Department of Urology, Shariati Hospital, Tehran University of Medical Sciences, Tehran, Iran

3 Institute for Urology and Reproductive Health, Sechenov University, Moscow, Russia

4 Department for Gynecology and Gynecologic Oncology, Gynecologic Cancer Unit, Comprehensive Cancer Centre, Medical University of Vienna, Vienna, Austria
5 Department of Urology, University Medical Center Hamburg-Eppendorf, Hamburg, Germany

6 Department of Urology, Jagiellonian University, Medical College, Cracow, Poland

7 Department of Urology, Weill Cornell Medical College, New York, NY, USA

8 Department of Urology, University of Texas Southwestern Medical Center, Dallas, TX, USA 